
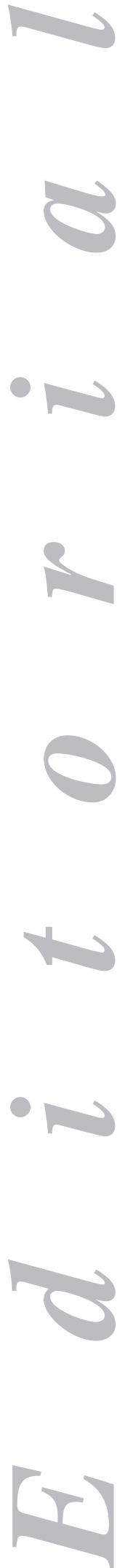

\section{Cholelithiasis and gastroesophageal reflux disease}

Gastroesophageal reflux disease has been connected with cholelithiasis for years. Saint's triad (1), a well-known association of hiatal hernia with cholelithiasis and colon diverticula that was much in fashion during the 1980's, represented a paradigmatic expression of westernization-induced gastrointestinal disturbances. In this issue of Revista Española de Enfermedades Digestivas, Pozo et al. (2) bring forward two of the components in that much debated Saint's triad: gastroesophageal reflux disease and cholelithiasis. The truth of the matter is that questions posed are many, and all of them entail an immediate clinical impact.

The first question is: is there really an association of cholelithiasis with gastroesophageal reflux disease? Should such an association exist beyond mere chance, then we should actively seek the accompanying condition in those who suffer from either of the aforementioned diseases, and provide a joint solution to this problem or, at least, consider the need to do so.

Both are common conditions in our country. Ten percent of the population has ultrasonographically demonstrated cholelithiasis (4), and $30 \%$ of the population suffers from reflux symptoms, $10 \%$ of these on a weekly basis (5). Chances are high that a patient with one condition will have the other simply because of their frequency; however, they are even higher when the fact that overweight is a risk factor for both is brought to our attention. If no association exists beyond mere chance (3), considering an active diagnostic approach in the absence of supporting clinical data is an unreasonable choice.

When both conditions coexist, therapeutic aspects are interesting. Statements such as "as he has these two things, let's operate and fix them both" or "while we are at it with this one, let's operate the other, it'll just take a few minutes more" are relatively commonplace. To which extent are these statements wise?

The mere coexistence of both conditions does not represent a combined surgical indication, and each of them should be individually assessed. Of course, if surgery is not indicated for either condition, its consideration in view of their association seems devoid of any logic. Therefore, the problem is broken down into two circumstances: should we perform a cholecystectomy when fundoplication is indicated? And viceversa: should we carry out a fundoplication when a cholecystectomy is to be performed? Surprisingly, the literature provides only few answers.

The performance of cholecystectomy during laparoscopic fundoplication is a topic that has been recently raised in the literature by the Mayo Clinic team at Jacksonville. With an extensive series of successive procedures, Klaus et al. (6) demonstrate that the performance of cholecystectomy in patients undergoing fundoplication results in no increased morbidity and no prolonged hospital stay, and has no significant impact on antireflux surgery outcomes. The authors recommend this approach, 
but the study's retropective nature and Ron Hinder's extensive experience in such procedures should lead us to carefully assess our setting and each subject before adopting such recommendation.

Not much information is there either on the reverse situation: should fundoplication be performed in patients where cholecystectomy is indicated? To find a study answering this question one must date back to 1980, when Stahlgreen et al. (7) showed the difference between performing a cholecystectomy and associating or not a fundoplication in those with demonstrated GERD. A considerable difference exists regarding morbidity, with 1 versus $8 \%$, respectively. Despite this, authors advise that fundoplication be performed in patients with GERD without waiting for medical therapy to fail, although the fact that medical treatment effectiveness was scarce during the 1970's weighed heavily on it (8).

In this issue of Revista Española de Enfermedades Digestivas (2), a team from Asturias' Central Hospital offers readers a study focused in the latter problem, which somehow updates results reported by Stahlgreen et al. by using the laparoscopic approach and basing the indication of associated fundoplication upon not only an objective study of GERD, but also inadequate symptom control from medical treatment. Results show that the addition of fundoplication prolongs (duplicates) surgery duration and slightly lengthens work resumption times (by almost one day), but is not associated with increased morbidity or hospital stay. Surgical team experience (no conversion to laparotomy in 442 procedures) is probably a decisive factor for such good results.

Should we recommend the combined procedure? A consequence of this study is that performing an associated fundoplication in patients with indicated cholecystectomy is a safe option provided the following two conditions are met: a clinical indication for fundoplication and the presence of an experienced surgical team. It is under such conditions that we should offer a patient undergoing cholecystectomy a choice to either stick to antireflux medical therapy or have fundoplication performed within the same surgical procedure.

E. Rey

Service of Digestive Diseases. Hospital Clínico San Carlos. Madrid, Spain

\section{References}

1. Burkitt DP, Walker ARP. Saint's triad: confirmation and explanation. S Afr Med J 1976; 50: 2136.

2. Pozo F, Giganto F, Rodrigo L. Colelitiasis no complicada asociada con ERGE: resultados de la cirugía laparoscopica combinada en pacientes con bajo riesgo quirúrgico. Rev Esp Enferm Dig 2004; 96 (4): 237-45.

3. Avidan B, Sonnenberg A, Schell TG, Sontag SJ. No association between gallstones and gastroesophageal reflux disease. Am J Gastroesnterol 2001; 96: 2858-62.

4. Martínez de Pancorbo C, Carballo F, Horcajo P, Aldeguer M, Villa I, Nieto E, et al. Prevalence and associated factorsfor gallstone disease: results of a population survey in Spain. J Clin Epidemiol 1997; 50: $1347-55$.

5. Díaz-Rubio M, Moreno-Elola-Olaso C, Rey E, Locke III GR, Rodríguez-Artalejo F. Symptoms of gastrooesophageal reflux: prevalence, severity, duration and associated factors in a Spanish population. Aliment Pharmacol Ther 2004; 19: 95-106.

6. Klaus A, Hinder RA, Swain J, Achem SR. Incidental cholecystectomy during laparoscopic surgery. Am Surg 2002; 68: 619-23.

7. Stahlgreen LH, Pagana TJ, Constantino GN. Funduplication for major reflux in patients with gallstones. Surg Gynecol Obst 1980; 150: 875-7.

8. Rey E, Díaz-Rubio M. Enfermedad por reflujo gastroesofágico: un siglo de historia. Rev Esp Enferm Dig 2003; 95 (extraordinario): 18-24. 


\section{Colelitiasis y enfermedad por reflujo gastroesofágico}

Desde hace años se ha puesto en relación la enfermedad por reflujo y la colelitiasis. La famosa triada de Saint (1) constituida por la asociación de hernia hiatal, colelitiasis y divertículos colónicos, muy en boga en los años 80, representaba una de las expresiones paradigmáticas de los problemas digestivos generados por la occidentalización. En este número de la Revista Española de Enfermedades Digestivas de Pozo y cols. (2) traen dos de las partes de la discutida (3) tríada de Saint: la enfermedad por reflujo gastroesofágico y la colelitiasis. Lo cierto es que son muchas las preguntas que surgen y todas con una repercusión clínica inmediata.

La primera cuestión es: ¿realmente existe una asociación entre colelitiasis y enfermedad por reflujo gastroesofágico? Si tal asociación existe más allá del puro azar deberíamos buscar activamente la otra enfermedad en aquellos que sufran una de ellas y proporcionar una solución conjunta al problema o, al menos, considerar la necesidad de hacerlo.

Ambos son problemas frecuentes en nuestro país. Un 10\% de la población tiene colelitiasis si se realiza una ecografía (4) y un 30\% de la población tiene síntomas de reflujo, un $10 \%$ de forma semanal (5). La probabilidad de que un paciente con uno de los dos problemas sufra el otro es alta simplemente por la frecuencia, pero mayor aún si consideramos que ambas tienen al sobrepeso como factor de riesgo. Si no existe una asociación más allá del azar (3), no es razonable pensar en una actitud diagnóstica activa en ausencia de datos clínicos que la dirijan.

$\mathrm{El}$ aspecto terapéutico cuando ambos problemas coexisten es interesante. Es relativamente frecuente escuchar afirmaciones como, "ya que tiene las dos cosas, le operamos y arreglamos ambos" o "ya que vamos a operar una, operemos la otra que total sólo son unos minutos más". Hasta qué punto estas afirmaciones son acertadas.

La mera coexistencia de ambos procesos no representa una indicación quirúrgica de tratamiento combinado y debemos evaluar singularmente cada una de ellas. Desde luego, si no existe indicación quirúrgica para ninguna de ellas, plantearse la necesidad de cirugía simplemente por la asociación de ambas parece carente de lógica. Por tanto, el problema se desglosa en dos circunstancias ¿debemos realizar una colecistectomía si se indica una funduplicatura? Y viceversa, ¿debemos realizar una funduplicatura si se va a realizar una colecistectomía? Sorprendentemente la literatura nos ofrece muy pocas respuestas.

La cuestión de realizar una colecistectomía durante una funduplicatura laparoscópica ha sido recientemente planteado en la literatura por el grupo de la Clínica Mayo de Jacksonville. Con una amplia serie de intervenciones consecutivas, Klaus y cols. (6) demuestran que la realización de una colecistectomía en pacientes a los que se realiza una funduplicatura no acarrea un aumento de la morbilidad, de la estancia hospitalaria ni tiene repercusiones relevantes sobre los resultados de la cirugía antirreflujo. Los autores recomiendan esta actitud, aunque la naturaleza retrospectiva del estudio y la enorme experiencia de Ron Hinder en estas intervenciones, nos deben inducir a evaluar nuestro medio y cada caso antes de aplicar esta recomendación.

Tampoco existe demasiada información respecto a la situación inversa: ¿se debe realizar una funduplicatura en pacientes con indicación de colecistectomía? Para en- 
contrar un estudio que responda esta pregunta hay que remontarse hasta 1980, donde Stahlgreen y cols. (7) muestran en una serie de pacientes consecutivos las diferencias entre realizar una colecistectomía y asociar una funduplicatura en aquellos que tras un estudio mostraron una ERGE. Hay una diferencia en la morbilidad considerable, con un 1 frente al 8\%, respectivamente. A pesar de ello, los autores aconsejan la realización de una funduplicatura en aquellos pacientes con ERGE sin esperar al fracaso del tratamiento médico, aunque probablemente pesaba mucho el hecho de la escasa eficacia del tratamiento médico en la década de los 70 (8).

En este número de la Revista Española de Enfermedades Digestivas (2), un grupo del Hospital Central de Asturias pone a nuestra disposición un estudio centrado en este último problema y que de alguna forma actualiza los resultados de Stahlgreen y cols., utilizando la vía laparoscópica y basando la indicación de funduplicatura asociada no sólo en el análisis objetivo de la ERGE, sino también con un control inadecuado de los síntomas con el tratamiento médico. Los resultados muestran que la adición de una funduplicatura aumenta el tiempo de quirófano (lo duplica) y prolonga ligeramente el tiempo de recuperación laboral (casi un día), pero no se asocia con una mayor morbilidad ni estancia hospitalaria. La experiencia del equipo quirúrgico (ninguna conversión a laparotomía en 442 intervenciones) probablemente es un factor decisivo en estos buenos resultados.

¿Debemos recomendar la intervención conjunta? La consecuencia de este estudio es que en pacientes con indicación de colecistectomía y bajo riesgo quirúrgico, la realización de una funduplicatura asociada es segura cuando se cumplan dos condiciones: indicación clínica para la funduplicatura y disponibilidad de un equipo quirúrgico experimentado. Es en estas condiciones cuando debemos ofrecerle al paciente que se va a someter a una colecistectomía entre seguir con tratamiento médico para el reflujo o asociar una funduplicatura en el mismo acto quirúrgico.

E. Rey

Servicio de Aparato Digestivo. Hospital Clínico San Carlos. Madrid 